Chapter 6

\title{
Dual-Wavelength Fiber Lasers for the Optical Generation of Microwave and Terahertz Radiation
}

\author{
Kavintheran Thambiratnam, Harith Ahmad and Mukul C. Paul \\ Additional information is available at the end of the chapter
}

http://dx.doi.org/10.5772/61690

\begin{abstract}
Dual-Wavelength Fiber Lasers (DWFLs), which provide a simple and cost-effective approach for the optical generation of Microwave $(\mathrm{MHz})$ and Terahertz $(\mathrm{THz})$ radiation. The emphasis of this review is to trace the early development of DWFLs, including the issues and limitations faced by the various gain media right to the latest advancements in this field as well as their roles in generating the desired output. This review covers both the simple approaches of narrow-band filters and comb filters for microwave radiation generation, as well as the use of DWFLs with diethylaminosulfurtetrafluoride or $\mathrm{LiNbO}_{3}$ crystals for generating $\mathrm{THz}$ radiation.
\end{abstract}

Keywords: Fibre Laser, Microwave, Terahertz, Dual-Wavelength

\section{Introduction}

The generation of Microwave (MHz) and Terahertz (THz) signals has been an area of intense research efforts due to the potential application for these signals in a multitude of applications. These new applications are spread across the board, encompassing conventional applications that are typically associated with $\mathrm{MHz}$ and $\mathrm{THz}$ signals such as communications and manufacturing [1-3] to novel and niche areas such as defence and science [4, 5].

While the generation of $\mathrm{MHz}$ and $\mathrm{THz}$ signals has been traditionally confined exclusively within the electromagnetic and radio frequency domains, recent developments in the field of photonics have made it possible for the generation of $\mathrm{MHz}$ and $\mathrm{THz}$ in this domain. This allows researchers to now overcome some of the technological limits on the development of these signals in the electromagnetic and radio frequency domains, and the same time allowing them to realize a vast new array of applications. Photonic based $\mathrm{MHz}$ systems could allow for 
the development of applications such as photonic generation, processing, control and distribution of $\mathrm{MHz}$ and millimeter-wave signals, while photonic based $\mathrm{THz}$ signal generation could in turns realize the development of new non-invasive sensing and measurement systems as well as expanding communications bandwidths. The interest in $\mathrm{MHz}$ based photonics systems is in the interactions between microwave and optical signals. The generation of microwave signals is typically a complicated affair, requiring expensive electronic circuits with multiple frequency doubling stages. This creates a major issue in terms of practically, and for safety purposes most microwave generation facilities are located at remote sites, thus making the distribution of $\mathrm{MHz}$ signals in the electrical domain a highly inefficient exercise as these signals will experience high losses during the transmission process. This is where photonic based MHZ systems has the significant advantage, as the generation of microwave signals in the optical domain alleviates these problems by allowing low-loss and broad bandwidth transmissions over optical fibres and at the same time taking advantage of the inherent immunity of optical fibres to electromagnetic waves. In most cases, microwave signals in the optical domain can be generated by the relatively simple process of optical heterodyning [6, 7], whereby the beating of two closely spaced but distinct frequencies results in the generation of the desired $\mathrm{MHz}$ output at a photodetector. Using this approach, $\mathrm{MHz}$ waves can be generated from a central office and distributed through an optical grid, thus simplifying equipment requirements and in turn reducing the cost and complexity of the system.

At the same time, intensive research has also been undertaken to investigate the field of $\mathrm{THz}$ radiation. $\mathrm{THz}$ radiation lies in the frequency gap between the infrared and microwave regions of the emission spectra, falling between the band gaps of $100 \mathrm{GHz}$ to $30 \mathrm{THz}$ and although long been eminent only in the domains of astronomy and analytical science, recent advances in technology have now seen its expansion into new areas of interest. This is due to the almost unlimited potential THZ radiation has for a wide variety of applications such as communications, security and defence, biological and medical sciences and spectroscopy to name a few. $\mathrm{THz}$ generation by optical sources typically entails non-linear optical frequency conversion, although initial research into this area was limited due to frequency and conversion efficiencies. However, with the availability of femtosecond lasers, $\mathrm{THz}$ frequencies and conversion efficiencies that could not be previously attained from optical sources can now be realized. Where the typical method of generating $\mathrm{THz}$ pulses would be by the use of femtosecond oscillators in the form of photoconductive switches and optical rectification, these pulses can now be realized using a variety of techniques that include the adaptation of femtosecond laser systems for generating of high intensity $\mathrm{THz}$ pulses as well as the exploitation of beats generated by two closely spaced lasing wavelengths. THz radiation is highly important for a multitude of applications, in particular the analysis of biological materials as the energy of $\mathrm{THz}$ photons is typically several orders of magnitude below the energy level required to ionize the valence electrons from biological molecules. This makes $\mathrm{THz}$ radiation a non-ionizing radiation, and gives it a fundamental advantage over ionizing radiation as it negates any harmful effects to biological structures that would result in the formation of highly reactive free radicals, which can cause secondary or indirect damage to other biomolecules. As such, $\mathrm{THz}$ radiation would find substantial potential for applications that require the analysis of biological matter, encompassing a wide range of effects from research such as spectroscopic analysis to real-world applications such as security applications. 


\section{Optical generation of microwave and $\mathrm{THz}$ radiation}

The generation of $\mathrm{THz}$ and microwave radiation by optical means can be typically accomplished by two methods. The first method entails the creation of an ultrafast photocurrent in a photoconductive switch or semiconductor using electric-field carrier acceleration, otherwise known as the photo-Dember effect. Alternatively, the desired radiation can be optically generated by non-linear optical effects such as optical rectification or optical parametric oscillations. Both these approaches are, however, rather difficult to achieve, requiring highly sensitive electronics or exotic media such as GaAs, GaSe, GaP, ZnTe, CdTe, diethylaminosulfurtetrafluoride (DAST) or $\mathrm{LiNbO}_{3}$ crystals.

Recently however, a new approach has shown much potential for the optical generation of $\mathrm{THz}$ radiation; optical heterodyning [6, 7]. By employing the optical heterodyning approach, both microwave and $\mathrm{THz}$ radiation can be generated as a result of the frequency differences between two closely spaced laser modes. When a photo-mixer with a short photo-carrier lifetime is exposed to these laser modes, the photocurrent is modulated at the frequency difference of the two laser modes, thereby generating an electromagnetic wave within the desired spectral range. The generated heterodyne beat can be represented mathematically as:

$$
\begin{aligned}
& E_{1}(t)=E_{01} \cos \left(\omega_{1} t+\phi_{1}\right) \\
& E_{2}(t)=E_{02} \cos \left(\omega_{2} t+\phi_{2}\right)
\end{aligned}
$$

With $\mathrm{E}_{01}$ and $\mathrm{E}_{02}$ representing the amplitude of the generated modes, while $\omega_{1}$ and $\omega_{2}$ representing the angular frequency of the same modes. The phases of the modes are given in (1) and (2) as $\phi_{1}$ and $\phi_{2}$ respectively, while $t$ denotes the time. Superimposing Equations (1) and (2) yields Equation (3), which is as follows:

$$
\mathrm{I}_{R F}=\mathrm{A} \cos \left[\left(\omega_{1}-\omega_{2}\right)+\left(\phi_{1}-\phi_{2}\right)\right]
$$

whereby $\mathrm{I}_{R F}$ represents the frequency of the generated electromagnetic wave and A being a constant determined by $\mathrm{E}_{01}$ and $\mathrm{E}_{02}$. Tuning the spacing between the two wavelengths will now yield the electromagnetic output in the desired spectrum, be it in the microwave or $\mathrm{THz}$ regions.

\section{MHz generation from DWFLs}

In a perfect scenario, the generation of $\mathrm{MHz}$ and $\mathrm{THz}$ radiation from DWFLs would require the interaction of two closely Single-Longitudinal Mode (SLM) beams propagating in the same 
laser cavity. An SLM output is desired, as the long cavities in most fiber laser configurations would typically lead to a dense group of wavelengths propagating alongside a central wavelength, in turn preventing the generation of a clean and stable output [8]. SLM outputs can be obtained from fibre lasers by employing either narrow band or comb filters. Narrow band filters encompass filters such as Fiber Bragg Gratings (FBGs) $[9,10]$ and arrayed waveguide gratings (AWGs), while comb filters encompass interferometers, such as the MachZehnder filter, Fabry-Perot filter and ring resonators. Both approaches have their advantages; narrow-band filters are easily integrated into laser cavities, allowing for the accurate filtering of wavelengths from a broad spectral band, while interferometers are easy to fabricate and provide uniform channel spacings. However, one would also need to consider the drawbacks of each approach; FBGs and AWGs are difficult to fabricate, whereas interferometers are usually very sensitive with substantial sizes and do not function well outside a laboratory environment.

The earliest, and arguably the easiest DWFL configuration for generating an SLM output would be the use of FBGs with two distinct but close filtering wavelengths to isolate the desired wavelengths from a broadband spectrum and allow only these two wavelengths to oscillate within the laser cavity. A typically example of this is seen in the configuration as proposed by D. S. Moon and Y. Chung [11], which uses a simple ring cavity with an Erbium Doped Fiber (EDF) acting both the broadband spectrum source and also the amplifier for the lasing wavelengths. The desired dual-wavelength output is achieved by integrating two FBGs into the laser cavity. Although the proposed design in simple, nevertheless it achieves its crucial objectives, which is to stable and uniform dual wavelength SLM output. The system as proposed in Figure 1 is highly stable, with a power fluctuation of less than $0.2 \mathrm{~dB}$ at room temperature and a relatively high Optical Signal to Noise Ratio (OSNR) of more than $60 \mathrm{~dB}$.

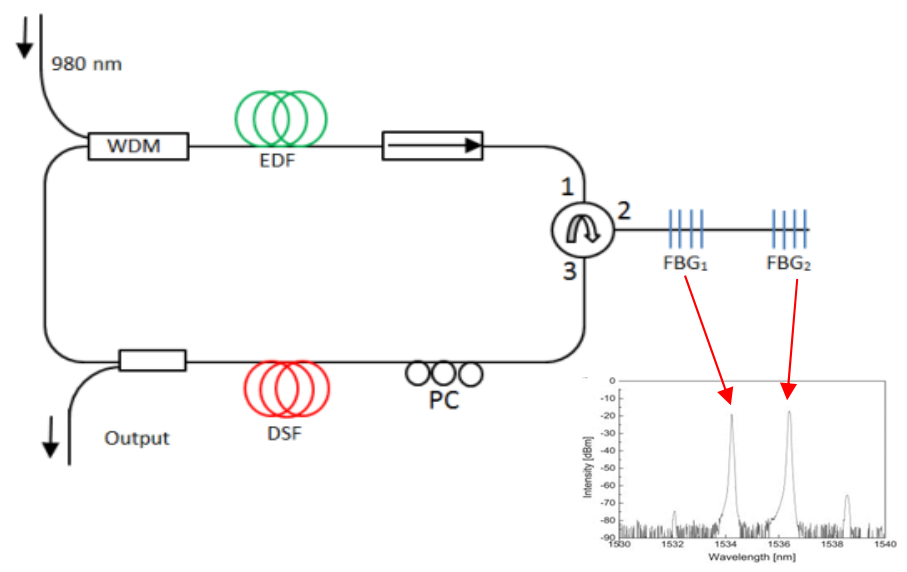

Figure 1. Experimental setup of a simple DWFL using an EDF as the gain medium. The inset of the figure shows the generated dual wavelength output obtained from the system [11]. Bear in mind though that the inset indicates where the dual-wavelength output is generated, not where the sampling is made. 
A particular problem arises though in the design of Figure 1, which revolves around the use of the EDF. Specifically, the issue that arises is that of homogenous broadening and cross-gain saturation, both of which are effects strongly felt in the EDF and will lead to a condition of unstable lasing. A straight-forward solution to this is to cool the EDF to sub-zero temperatures, typically around $77 \mathrm{~K}$ using liquid nitrogen. While this alleviates the effects of homogenous broadening and cross-gain saturation, it is for all purposes impractical and cannot be practically realized. Alternatively, exotic EDFs can also be used, such as those with specially designed structures or by taking advantage of various optical effects such as Polarization Hole Burning (PHB). Again, these may allow the desired goals to be achieved, but also increases the cost and complexity of the setup. The authors of [11] opted for a different approach, which is to suppress the line-broadening using a $2 \mathrm{~km}$ long Dispersion Shifted Fiber (DSF). While this approach was successful in creating the desired SLM output, careful balancing is required when using the DSF, thus adding a certain level of complexity to the system.

As a result of the effects of homogenous line broadening, in-homogenous gain media has also been investigated for the development of a stable, dual-wavelength SLM output. Among the successful demonstrations of this approach was that by X. Chen et al. [12] who were able to use two Ultranarrow Transmission Bands (UNTB) FBGs in conjunction with a Semiconductor Optical Amplifier (SOA) to generate the desired output for operation in the microwave regime. The key advantage of this setup, as shown in Figure 2 is the use of the SOA as the gain medium which is has a very much lower homogeneous line-broadening effect as compared to the EDF.

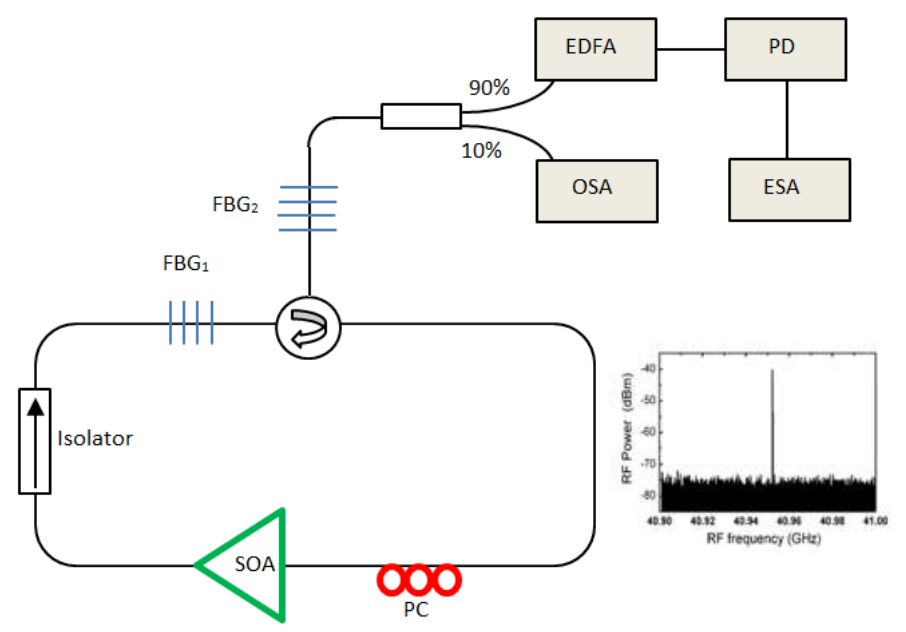

Figure 2. SLM dual wavelength fiber laser utilizing two UTNB FBGs. The mixing of the two wavelengths results in the generation of a microwave signal as seen from a radio frequency spectrum analyser, as shown in the figure [12].

As a result, the effects that typically arise in homogenously broadened gain media, such as mode-competition is negligible, and thus the two SLM wavelengths are able to lase in the same cavity with the relative phase fluctuations between the two wavelengths being low and thus 
allowing the system to generate a low-phase-noise microwave signal without requiring a microwave reference source. This particular work was able to generate microwave signals at $40.95,18.68$ and $6.95 \mathrm{GHz}$, corresponding to the channel spacings of the three different dualwavelength UNTB FBGs incorporated into the laser cavity. A spectral width as small as $80 \mathrm{kHz}$ was with a frequency stability better than $1 \mathrm{MHz}$ when operating at room temperature in a free-running mode.

While the use of the SOA resolved the issues related to the stability of the lasing wavelengths, improved performance in terms of the channel spacing and thus, the frequencies of the generated microwave and $\mathrm{THz}$ radiation outputs could be achieved by using other wavelength filtering mechanisms in place of the conventional FBGs, such as Phase-Shifted FBGs (PSFBGs) [13], Inverse-Gaussian Apodized FBGs (IGAFBGs) [14] and Chirped Moire FBGs (CMFBG) [15]. The advantages of these approaches has already been proven, such as the wider range of the microwave signals generated as reported by E. Guillermo et al. [13]. E. Guillermo proposed and demonstrated a setup utilizing two cascaded PSFBGs written onto FBGs and configured to form a distributed feedback fiber laser. Using the PSFBGs, a dual-wavelength laser output is achieved with each wavelength having a Full-Width at Half Maximum (FWHM) linewidth of $0.22 \mathrm{pm}$ in the single-wavelength regime. Piezoelectric actuators were utilized as the tuning mechanism, thus giving the user dynamic control over the wavelength spacing between the two lasing modes. A continuous tuning range of between 5 to $724 \mathrm{pm}$ of was obtained, giving an equivalent microwave frequency range of $0.72 \mathrm{GHz}$ to $92 \mathrm{GHz}$.

While the generation of the dual-wavelength SLM outputs was made possible by the FBG, real-world applications would demand a more consistent and uniform mechanism of obtaining these wavelengths. In this regard, the FBG falls short, as each time the system is deployed, fine tuning of the FBG's operating wavelengths and other such parameters needs to be made to ensure that the desired result is obtained. As such, focus on the development of the DWFL now shifted towards new wavelength filtering mechanisms that would give the same output regardless of the environment it was operating in. In this manner, a viable solution was found in the form of the Arrayed Waveguide Grating (AWG). When employed in a laser cavity, the AWG allowed for the quick and easy manipulation of the spacing between two oscillating wavelengths simply by selecting different channels on the AWG. As the channel spacings on the AWG were constant, thus a uniform output could be realized. Figure 3 shows such a system, which utilizes two AWGs to generate the desired wavelength spacings. This reliable yet simple approach was demonstrated by $\mathrm{H}$. Ahmad, et. al. [16, 17] and used a $1 \mathrm{~m}$ long highly doped Leikki Er80-8/125 EDF as the linear gain medium. Two AWGs were used as the narrowband filters. Similar to the aforementioned approach, the suppression of unwanted modes is carried out using a $7 \mathrm{~cm}$ long un-pumped Leikki Er80-8/125 EDF and sub-ring cavity. An output is highly stable output is obtained, with close channel spacings of between 0.01 to 0.03 $\mathrm{nm}$ and beating frequencies of between 1.4 to $3.2 \mathrm{GHz}$. The addition of a Mach-Zehnder modulator, driven at $180 \mathrm{kHz}$ in the above setup adds the ability to obtain a distinct oscillating wavelength in addition to the dual-wavelength output should the need arise.

In addition to narrow-band filtering, the use of comb filters as a means to obtain a dualwavelength output has also become widely accepted. One of the better examples of this 
approach is that of the reconfigurable dual-pass Mach-Zehnder Interferometer (MZI) filter, that was demonstrated by F. Wang et al. [18]. In this approach, the dual-pass MZI is incorporated into an EDF based ring fiber laser with a cavity that has a high extinction ratio, as shown in Figure 4.

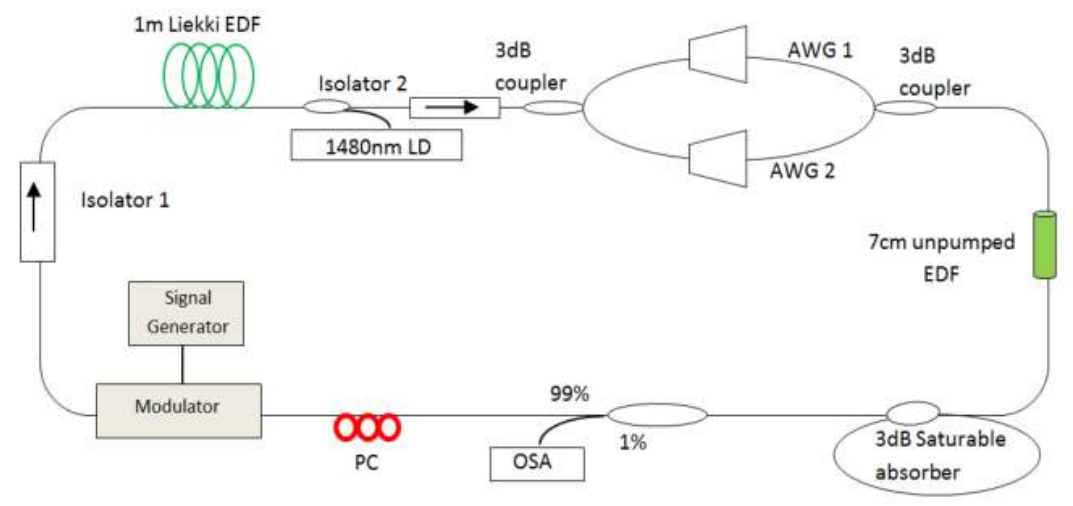

Figure 3. Experimental setup of AWG based dual wavelength SLM fiber laser (above) and the resulting microwave outputs with their corresponding dual-wavelength outputs [16].

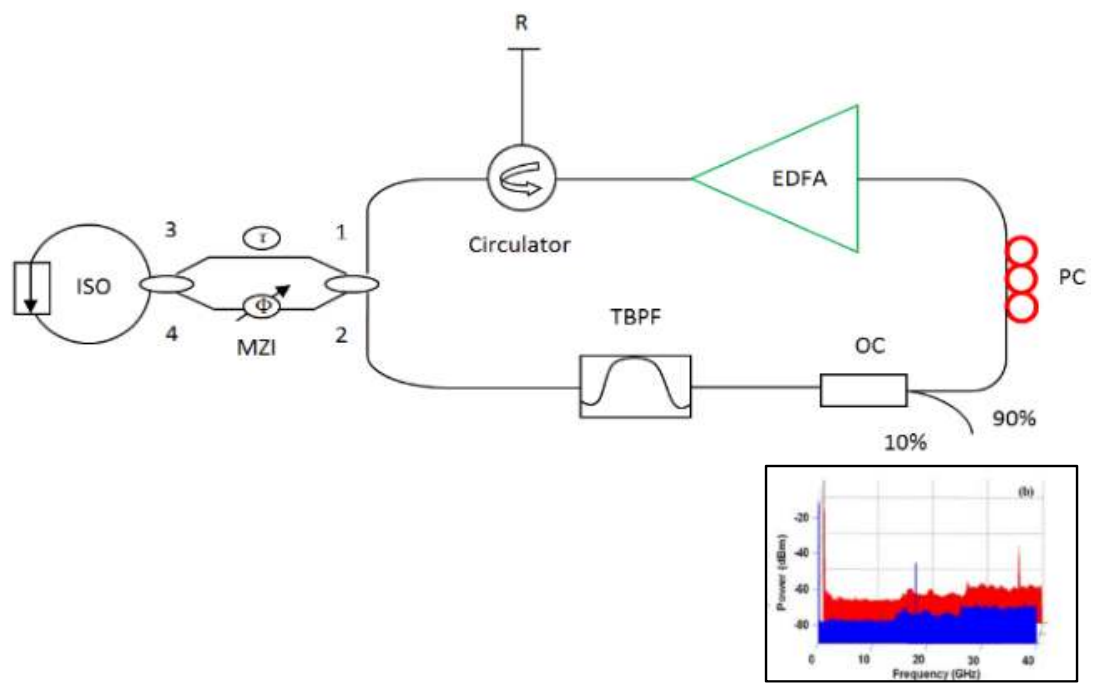

Figure 4. Experimental setup of the dual-pass MZI filter based dual-wavelength SLM fiber laser (above). The resulting microwave outputs are shown also shown in the figure (below), with the initial output shown in the forward spectrum, while the second microwave output, obtained after the channel spacing has been tuned, is shown in the spectrum at the back [18]. 
The proposed setup allows for the generation of a microwave output at signal frequencies of 9.76 GHz, $17.78 \mathrm{GHz}$ and $35.67 \mathrm{GHz}$, although other frequencies can also be realized. The optical spectra of the system can be tuned from 1530 to $15675 \mathrm{~nm}$ using the Tunable Bandpass Filter (TBPF) inside the laser cavity. S. Pan and J. Yao on the other hand explored a different approach to produce a novel DWFL capable of generating an SLM output from an EDF laser. Their approach was based on a sigma architecture consisting of a ring loop and a linear standing wave arm [8] as illustrated in Figure 5. This approach is highly advantageous as it prevents gain competition among the oscillating wavelengths by placing the gain medium in the standing-wave arm and introducing the PHB effect into the system by the polarization multiplexing of the two lasing wavelengths in the ring loop of the cavity.

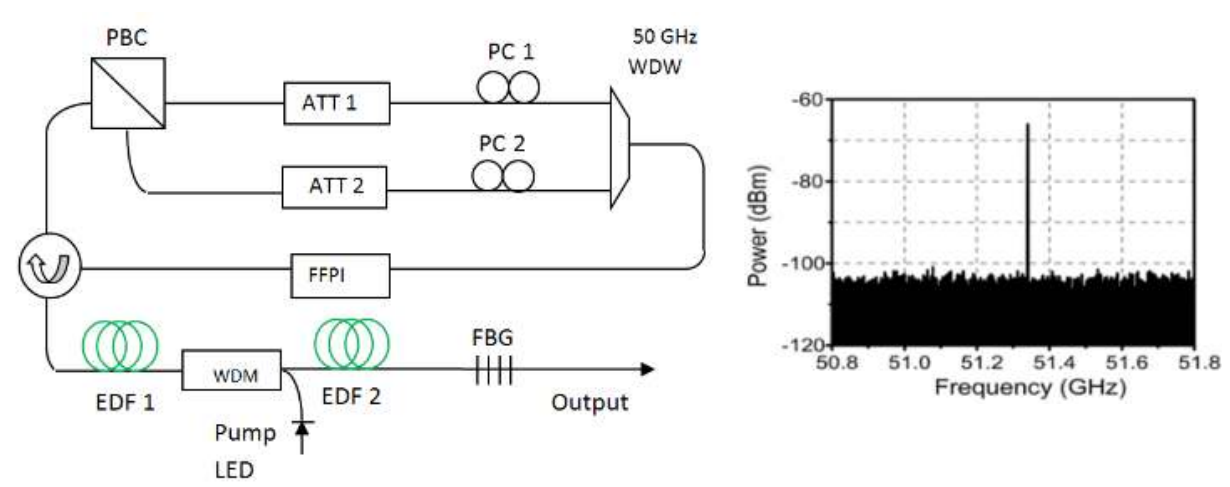

Figure 5. Experimental setup utilizing Fabry-Perot interferometer as SLM dual wavelength fiber laser. A single microwave output at approximately $51.3 \mathrm{GHz}$ is obtained from the system [8].

This provides a stable output, and by integrating an ultranarrow Fabry- Perot Filter (FPF) into the cavity in the form of an un-pumped EDF, SLM operation can be realized. As a Lyot filter is formed for each wavelength, thus wavelength switching can be achieved by simply adjusting the polarization state of either wavelength. The proposed system was able to generate a microwave frequency output in a tunable range of $\sim 10$ to $\sim 50 \mathrm{GHz}$.

Recently, a new approach towards achieving a DWFL with an SLM has been demonstrated using a ring resonator. This approach, as proposed by Y. Zhang et. al. successfully demonstrated a tunable SLM dual wavelength fiber laser based on a microwave photonic notch filter using a microfiber ring resonator [19] as shown in Figure 6.

In this experiment, the microfiber ring resonator (MRR) acts as a comb filter, while an unpumped Polarization Maintaining EDF (PM-EDF) with an Au film coated at the end surface allows for the suppression of the mode competition and control of SLM output. A wavelength spacing of $1.02 \mathrm{~nm}, 0.82 \mathrm{~nm}$, and $0.65 \mathrm{~nm}$ is reported by adjusting the MRR diameter, giving an equivalent microwave signal at $2.31 \mathrm{GHz}$ with an OSNR of $35 \mathrm{~dB}$. Similar results have also been obtained using microfiber couplers [20]. 


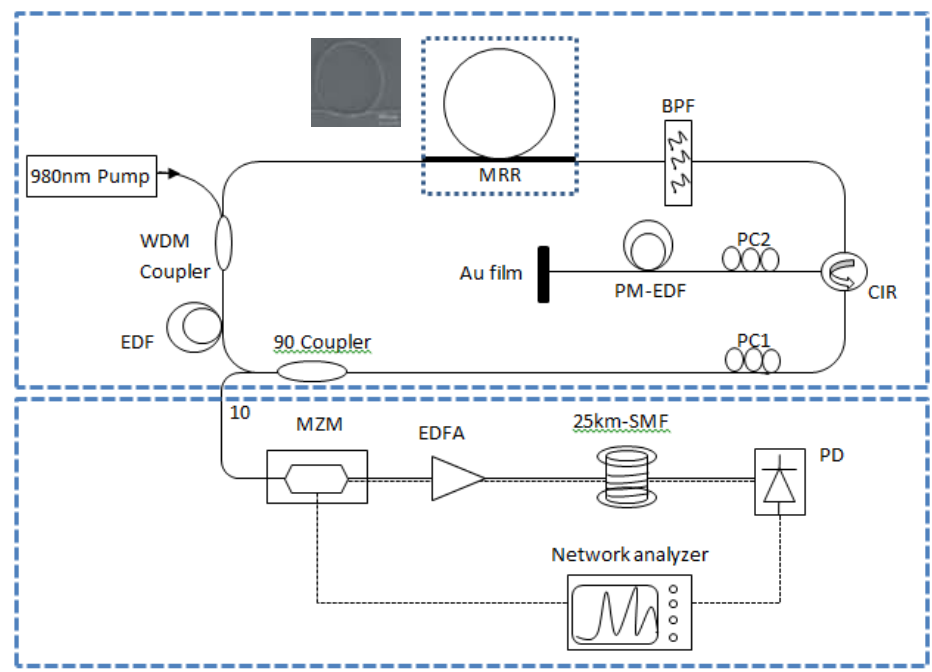

Figure 6. Experimental setup of the micro-ring resonator for dual-wavelength SLM generation. The inset shows the actual resonator ring [19].

\section{Terahertz generation from dual wavelength fiber lasers}

While microwave frequencies can be easy to generate, the optical generation of $\mathrm{THz}$ radiation can be much more complex. Unlike microwave radiation, the optical generation of $\mathrm{THz}$ radiation requires dual-wavelength near-Infrared (IR) laser fields to stimulate nonlinear polarizations in bulk Nonlinear Optical (NLO) crystals. Under suitable phase matching conditions, second order nonlinearity induced parametric process can lead to the generation of coherent $\mathrm{THz}$ radiation. However, due to the inherent characteristics of ultra-wideband $\mathrm{THz}$ frequency tunability (from sub-THz to tens of THz) that arise from the NLO crystals, Difference Frequency Generation (DFG) THz sources are very attractive for a wide range of applications in high-resolution spectroscopy and imaging. Traditionally, DFG THz sources were obtained using a dual wavelength approach by independent sources, although this method can be complex in terms of the necessary mechanical alignment and also the fine adjustment required in obtaining the spatial overlap of laser transverse modes [21, 22]. Alternatively, Optical Parametric Oscillators (OPO) can also be employed for this purpose, but although they can provide an output with a high power and also wide tenability, they are large, difficult to align and in most cases very expensive $[23,24]$. As such, DWFLs are now being seen as a viable alternative to obtain the necessary wavelengths from a fiber laser for $\mathrm{THz}$ radiation generation, while at the same time leveraging on the robustness, compactness and stability of the fiber laser.

One of the most reliable approaches towards obtaining the desired frequency output is the use of a wavelength selector and laser oscillation, provided by linearly Chirped Fibre Braggs 
Gratings (CFBGs) and SOAs. A system, as proposed by M. Tang et. al. [25] takes advantage of a thermo-optical phase change that allows seamless wavelength tuning; a single longitudinal mode operation is capable of achieving Continuous Wave (CW) dual-wavelength fibre laser with emissions at approximately $1060 \mathrm{~nm}$ using a Polarization Maintaining Ytterbium Fibre Amplifier (PM-YDFA) to amplifies the emission of the laser in order to pump a NLO DAST crystal. This setup is shown in Figure 8.

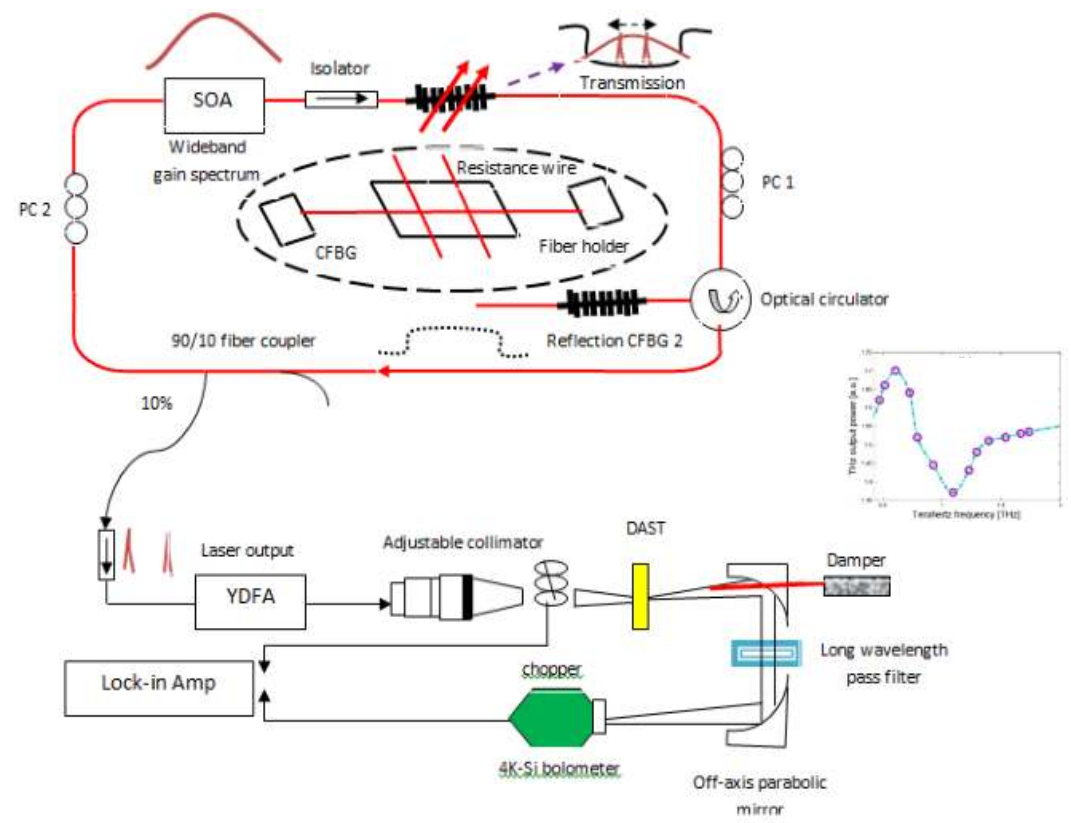

Figure 7. Setup of the DWFL for THz generation (above) and the resulting THz output that can be obtained from the system (below) [25].

The upper half of Figure 9 represents the ring configuration DWFL, which is based on a gain medium consisting of a fibre pig-tailed wideband $1060 \mathrm{~nm} \mathrm{SOA}$. This setup allows for a high saturation output power of $18 \mathrm{dBm}$ and a wide optical bandwidth of $60 \mathrm{~nm}$ with $+15 \mathrm{~dB}$ gain to be realized, with a relatively compact form factor as the ring cavity length is only $11.2 \mathrm{~m}$ long. The longitudinal mode spacing is $17.86 \mathrm{MHz}$, while unidirectional ring cavity and the stability of laser oscillations is maintained by means of optical isolator and polarization controllers at PC-1 and PC-2 respectively. The latter permits the laser polarization state to be optimized during propagation for the lasing and subsequently in amplification operations. The Two CFBGs, CFBG1 and CFBG2 are integrated into the cavity. CFBG1, which is $2.5 \mathrm{~cm}$ long is spliced into the cavity for transmission modes, and an identical to CFBG2 that is used for reflection alongside a 3-port optical circulator. Both CFBGs have a linear chirp rate of 4.5 $\mathrm{nm} / \mathrm{cm}$, which gives a reflection / stop band of approximately $10 \mathrm{~nm}$. The inscription of the linearly chirped CFBG is achieved using hydrogen-load Nufern PS1060 photosensitive fibre with the ultraviolet scanning beam at 1060nm wavelength. 
The two NiCr resistance wires shown in the figure are perpendicular to CFBG1, and lie across a heat sink while at the same time making contact with the fibre at two points. These wires have a cross-section diameter of $150 \mu \mathrm{m}$ and are powered by a DC power supply to act as heating elements. As the refractive index of the silica glass fibre is temperature dependent, thus the points of the fibre in contact with heated elements will cause a relative phase change in a passing optical signal. This thermo-induced phase change becomes sufficiently large to break the linear chirp relationship at the CFBG, which results in a sharp transmission rise in the stop-band region of the CFBG. Two transmission peaks can occur simultaneously by means of mechanical operation on the heating elements. Using the DAST crystal, this particular DWFL setup is able to successfully generate $\mathrm{THz}$ radiation in the $\mathrm{CW}$ regime from 0.5 to $2 \mathrm{THz}$. The $\mathrm{SOA}$ as gain medium is able to suppress gain competition and any noise that arises from laser beating, while the CFBG wave selector efficiently controls the frequency difference of dualwavelength single longitudinal mode lasing operations near $1060 \mathrm{~nm}$. The thermo-optical induced phase changes lead to very sharp transmission peaks, and two mechanically adjustable fibre contact positions allow for two simultaneous wavelength oscillations.

The optical generation of microwave and $\mathrm{THz}$ radiation will undoubtedly open up a whole new aspect of research and development due to the immense potential these systems have on a variety of applications. Although this chapter has been able to give an overview of the developments of the DWFL that has led to today's technologies for microwave and THz radiation generation, it far from all-encompassing. With the continued pace of development and growing interest in this area, there is no doubt that this field will continue to grow and generate many interesting outputs in the times to come.

\section{Author details}

Kavintheran Thambiratnam ${ }^{1 *}$, Harith $\mathrm{Ahmad}^{2}$ and Mukul C. Paul ${ }^{3}$

*Address all correspondence to: kavintheran@gmail.com

1 Malaysian Industry Government Group for High Technology (MiGHT), Malaysia

2 Photonics Research Centre, Malaysia

3 Central Glass and Ceramic Research Institute, India

\section{References}

[1] J. Kim, F. X. Kärtner. (2010, June). Microwave signal extraction from femtosecond modelocked lasers with attosecond relative timing drift. Opt. Lett. [Online]. 35(12), pp. 2022-2024. Available: http://dx.doi.org/10.1364/OL.35.002022 
[2] E. I. Ackerman and A. S. Daryoush. (1997, Aug). Broad-band external modulation fiber-optic links for antenna-remoting applications. IEEE Transactions on Microwave Theory and Techniques. [Online]. 45(8), pp. 1436-1442. Available: 10.1109/22.618449

[3] S. A. Pappert, C. K. Sun, R. J. Orazi, T. E. Weiner. (2000, May). Microwave fiber optic links for shipboard antenna applications. Presented at IEEE International Conference on Phased Array Systems and Technology, Proceedings. [Online]. pp. 345-348. Available: 10.1109/PAST.2000.858971

[4] A. S. Daryyoush. (2002, September). Microwave Photonics in Dual-Use Military Systems - A Personal Perspective. Presented at the RTO SET Lecture Series on "Optics Microwave Interactions", held in Jouy en Josas, France, 2-3 September 2002; Duisburg, Germany, 5-6 September 2002; Budapest, Hungary, 9-10 September 2002. [Online]. Available: http://ftp.rta.nato.int/public/PubFullText/RTO/EN/RTO-EN-028/ EN-028-\$I.pdf]

[5] J. Yao. (2009, Feb.). Microwave Photonics. Journal of Lightwave Technology. [Online]. 27(3), pp. 314-335. Available: 10.1109/JLT.2008.2009551

[6] J. J. Pan. (1989, Jan). Cost-Effective Microwave Fiberoptic Links Using The Heterodyne Laser. Proc. SPIE 0995, High Frequency Analog Communications. [Online]. 0995, pp. 94-98, Availbale: doi:10.1117/12.960149; http://dx.doi.org/10.1117/12.960149

[7] J. Genest, M. Chamberland, P. Tremblay, M. Tetu. (1997, Jun). Microwave signals generated by optical heterodyne between injection-locked semiconductor lasers. IEEE Journal of Quantum Electronics. [Online]. 33(6), pp. 989-998. Available: $10.1109 / 3.585487$

[8] S. Pan, and J. Yao. (2009, March). Frequency-switchable microwave generation based on a dual-wavelength single-longitudinal-mode fiber laser incorporating a high-finesse ring filter. Optics Express. [Online]. 17(14), pp. 12167-12173. Available: http:// dx.doi.org/10.1364/OE.17.005414

[9] L. Bo, T. Swee Chuan, J. Meng, P. Shum. (2011, September). Dual-wavelength fiber laser using an inverse-Gaussian apodized fiber Bragg grating for tunable microwave generation. Presented at The 16th Opto-Electronics And Communications Conference, OECC 2011, Taiwan. [Online]. pp. 196-197. Available: http://dx.doi.org/ 10.1364/AO.50.004912

[10] [10] X. Liu, X. Yang, F. Lu, J. Ng, X. Zhou, C. Lu. (2005, Jan). Stable and uniform dualwavelength erbium-doped fiber laser based on fiber Bragg gratings and photonic crystal fiber. Optics Express. [Online]. 13(1), pp. 142-147. Available: http://dx.doi.org/ 10.1364/OPEX.13.000142

[11] [11] D. S. Moon, and Y. Chung. (2013, Jan). Switchable dual-wavelength erbium-doped fiber ring laser assisted with four-wave mixing of dispersion-shifted fiber. Optics Communications, 286(1), pp. 239-243. 
[12] [12] C. Xiangfei, D. Zhichao, and Y. Jianping. (2006, Feb). Photonic generation of microwave signal using a dual-wavelength single-longitudinal-mode fiber ring laser. IEEE Transactions Microwave Theory and Techniques. [Online]. 54(2), pp. 804-809. Available: 10.1109/TMTT.2005.863064

[13] G. E. Villanuevaa, J. Palacía, J. L. Cruzb, M. V. Andrésb, J. Martía, P. Pérez-Millán. (2010, December). High frequency microwave signal generation using dual-wavelength emission of cascaded DFB fiber lasers with wavelength spacing tenability. Optics Communications. [Online]. 283(24), pp. 5165-5168. Available: http:// www.sciencedirect.com/science/article/pii/S0030401810008357

[14] B. Lin, S. C. Tjin, M. Jiang, P. P. Shum, Y. He, Y. Ge. (2011, July). Dual-wavelength fiber laser using an inverse-Gaussian apodized fiber Bragg grating for tunable microwave generation. Presented at The 16th Opto-Electronics and Communications Conference, OECC 2011, Taiwan. [Online]. pp. 196-197. Available: http:// ieeexplore.ieee.org/xpl/abstractAuthors.jsp?arnumber=6015080

[15] S. Feng, S. Lub, W. Peng, Q. Lia, C. Qia, T. Fenga, S. Jian. (2013, February). Photonic generation of microwave signal using a dual-wavelength erbium-doped fiber ring laser with CMFBG filter and saturable absorber. Optics $\mathcal{E}$ Laser Technology. [Online]. 45, pp. 32-36. Available: http://www.sciencedirect.com/science/article/pii/ S0030399212003532

[16] H. Ahmad, A. A. Latif, J. M. Taib, S. W. Harun. (2013). Tunable, low frequency microwave generation from AWG based closely-spaced dual-wavelength single-longitudinal-mode fibre laser. J. Europ. Opt. Soc. Rap. Public. [Online]. 8(13038), Available: 10.2971/jeos.2013.13038

[17] [17]H. Ahmad, M. Z. Zulkifli, A. A. Latif, S. W. Harun. (2009, December). Tunable Dual Wavelength Fiber laser Incorporating AWG and Optical Channel Selector by Controlling the Cavity Loss. Opt. Comm. [Online]. 282(24), pp. 4771-4775. Available: http://www.sciencedirect.com/science/article/pii/S003040180900844X

[18] F. Wang, X. Zhang, Y. Zhang, E. Xu. (2011, January). A Tunable and Switchable Single-longitudinal-mode Dual-wavelength Fiber Laser for Microwave Generation. Proc. SPIE 7987, Optoelectronic Materials and Devices V. [Online]. 7987, Available: doi: $10.1117 / 12.888113$

[19] [19] Z. Yu, Z. X. Liang, C. G. Jie, X. E. Ming, H. D. Xiu. (2010, July). A Microwave Photonic Notch Filter Using a Microfiber Ring Resonator. Chin. Phys. Lett. [Online]. 27(7), pp. 74207-074207. Available: http://iopscience.iop.org/0256-307X/27/7/074207

[20] [20]A. Sulaiman, S. W. Harun, M. Z. Muhammad, H. Ahmad (2013, July). Compact Dual-Wavelength Laser Generation using Highly Concentrated Erbium-Doped Fiber Loop Attached to Microfiber Coupler. IEEE J. of Quant. Electron. [Online]. 49(7), pp. 586-588. Available: http://ieeexplore.ieee.org/xpl/articleDetails.jsp?arnumber $=6512030$ 
[21] [21] A. Klehr, J. Fricke, A. Knauer, G. Erbert, M. Walther, R. Wilk, M. Mikulics, M. Koch. (2008, April). High-power monolithic two-mode DFB laser diodes for the generation of THz radiation. IEEE J. Sel. Top. Quant. Electron. [Online]. 14(2), pp. 289-294. Available: 10.1109/JSTQE.2007.913119

[22] N. J. Kim, Y. A. Leem, J. H. Shin, C. W. Lee, S. P. Han, M. Y. Jeon, D. H. Lee, D. S. Yee, S. K. Noh, and K. H. Park. (2010, September). Widely tunable dual-mode multisection laser diode for continuous-wave THz generation. Presented at 35th international conference on infrared, millimeter and terahertz waves (IRMMW-THz), We-C3.1, Rome. [Online]. pp. 1-2. Available: 10.1109/ICIMW.2010.5612426

[23] [23] A. Godard, M. Raybaut, O. Lambert, J.-P. Faleni, M. Lefebvre, E. Rosencher. (2005, September). Cross-resonant optical parametric oscillators: study of and application to difference-frequency generation. J. Opt. Soc. Am. B. [Online]. 22(9), pp. 19661978. Available: http://www.opticsinfobase.org/josab/abstract.cfm?URI=josab-22-9-1966

[24] K. Kawase, T. Hatanaka, H. Takahashi, K. Nakamura, T. Taniuchi, H. Ito. (2000, December). Tunable terahertz-wave generation from DAST crystal by dual signal-wave parametric oscillation of periodically poled lithium niobate. Opt. Lett. [Online]. 25(23), pp. 1714-1716. Available: http://dx.doi.org/10.1364/OL.25.001714

[25] M. Tang, H. Minamide, Y. Wang, T. Notake, S. Ohno, H. Ito. (2011, January). Tunable terahertz-wave generation from DAST crystal pumped by a monolithic dual-wavelength fiber laser. Opt. Express [Online]. 19(2), pp. 779-786. Available: http:// dx.doi.org/10.1364/OE.19.000779. 